\title{
NOTAS TAXONÓMICAS EN ASCLEPIADOIDEAE (APOCYNACEAE)
}

\author{
JORGE FONTELLA PEREIRA1 ${ }^{1,2}$, RENATA G. P. SANTOS ${ }^{1}$ y SERGIO A. CÁCERES MORAL ${ }^{3}$
}

\begin{abstract}
Summary: Taxonomical notes in Asclepiadoideae (Apocynaceae). A new combination, two new typifications, and six new synonyms are here established for the flora of the Southern Cone.
\end{abstract}

Key words: Apocynaceae, Asclepiadoideae, Nomenclature, Southern Cone, Taxonomy.

Resumen: Una nueva combinación, dos nuevas tipificaciones y seis nuevos sinónimos son aquí establecidos para la flora del Cono Sur.

Palabras clave: Apocynaceae, Asclepiadoideae, Cono Sur, Nomenclatura, Taxonomía.

\section{INTRODUCCIÓN}

Durante la p reparación de la $\mathrm{Su}$ bfamilia Asclepiadoideae (Apocynaceae) para su publicación en la Flora del Paraguay, hemos encontrado la necesidad de lectotipif icar dos nombres, establecer tres nuevos sinónimos, hacer una nueva combinación y me ncionar nuevos registros para Argentina y Paraguay.

Rojasia bornmuelleri (Schltr. ex Malme) Fontella, S. C áceres \& R. Santos, comb. nov. Gyrostelma bornmuelleri Schltr. ex Malme, Ark. Bot. 25A(7): 12. 1932. Tipo: ARGENTINA. Misiones, Santa Ana, A. de Llamas 4866 (Lectotipo S! aquí designado; sintipo Bornmuelleri 2999, JE!).

1 Departamento de Botânica do Museu Nacional Universidade Federal do Rio de Janeiro (UFRJ), Horto Botânico, Quinta da Boa Vista s.n., S. Cristóvão, Rio de Janeiro, RJ, Brasil. E-mail: jofope@mn.ufrj.br

${ }^{2}$ Conselho Nacional de Desenvolvimento Científico, Brasil (CNPq) E-mail: natagabrielle@yahoo.com.br

Instituto de Botánica del Nordeste, IBONE-CONICET.

Facultad de Ciencias Agrarias, Universidad Nacional del

Nordeste, FCA-UNNE. Casilla de Correo 209, 3400

Corrientes, Argentina. E-mail: ibonebiblio@agr.unne.edu.ar
En el protólogo, el material tipo está indicado de la siguiente manera: "Brasil: Rio Grande do Sul, Neu Württemberg, Estancia L. Gomez, 18.X.1904 (J. Bornmüller n. 299). A rgentina: Misiones, Santa Ana, X.1901 (A. de L lamas n. 4866)". Entre estos dos sintipos de JE y $\mathrm{S}$ resp ectivamente, se seleccionó el ejemplar de L lamas 4866 como lectotipo, por concordar mejor con los datos del protólogo y por haber sido examinado por Malme en el herbario de Es tocolmo donde este botánico trabajó.

Gyrostelma bornmuelleri fue descrita inicialmente por Malme (1932) y redescrita posteriormente por J. Bornmueller (1934); Meyer (1970) la ilustró por primera vez para Argentina.

Para el tratam iento de es ta especie seguimos el concepto genérico de Goyder (2006) que considera al género Rojasia Malme distinto a Matelea Aubl. La especie aquí tratada pres enta los caracteres diagnósticos del género Rojasia, como el h ábito decumbente, tallo muy ramificado e in florescencias parciales en cimas umbeliformes subaxilares, opuestas y alternas, laxas, pedunculadas, flores con pedicelos largos, lóbulos de la corola larguísimos en relación con el tubo y caudículas filiformes insertas en la parte media o en el tercio superior de 1 os polinios. Gyrostelma descripto por Fournier en 1885 
(género con una sola especie, Gyrostelma oxypetaloides), se caracteriza principalmente por el hábito erecto, tallo sin o con pocas ramificaciones, inflorescencias parciales en cimas densas, umbeliformes, subaxilares y alternas, sésiles, flores subsésiles, lóbulos de la corola no tan largos como el tubo y caudículas más largas, insertas en el tercio superior de los polinios.

De acuerdo con Ezcurra et al. (2008), Gyrostelma (=Rojasia bornmuelleri) vive en Argentina (Corrientes y Misiones) y Brasil (Paraná y Rio Grande do Sul), y ahora es registrada por primera vez para Paraguay. Habita ambientes soleados, desde campos secos limpios a campos altos en regiones montañosas o próximos a bosques. Morillo (1984) transfirió Gyrostelma oxypetaloides E. Fourn. a Matelea Aublet (1775), pero nosotros preferimos mantener a Gyrostelma E. Fourn. como un género distinto del taxón descripto por Aublet (loc. cit.), como lo indica Rapini (2001).

Material estudiado: BRASIL. Paraná: Guarapuava, Entre Ríos, 21-X-1969, Hatschbach 22579 (MBM, CTES); Rio Grande do Sul: Giruá, Granja Sodol, 08XI-1966, Hagelund 4828 (CTES). PARAGUAY. Misiones: $12 \mathrm{~km}$ de San Ignacio, camino a Pilar, 15XI-1978, Arbo et al. 1818 (CTES). ARGENTINA. Corrientes: Santo Tomé, $8 \mathrm{~km}$ E de Gobernador Virasoro, camino a Garruchos, 5-XII-1970, Krapovikas et al. 17117 (CTES); ibidem: Ituzaingó, Ruta 12,2 km antes del límite con Misiones, 11-IX1978, Cristóbal \& Krapovikas 1789 (CTES).

Orthosia virgata (Poir.) E. Fourn., Mart., Fl. Bras. 6(4): 223. 1885.

Periploca virgata Poir., Syst. Veg. 192. 1804. Tipo: Cette plant croît dans lê Indes orientales, h (v. s. in herb. Lam., holotipo P!).

Metastelma virgatum (Poir.) Decne., Prodr. 8: 515. 1844.

Ionidium diffusum Gillies ex Hook. \& Arn., Bot. Misc. 3: 145. 1833. Tipo: ARGENTINA. Buenos Ayres, Dr. Gillies s.n. (K)

Cynanchum diffusum (Gillies ex Hook. \& Arn.) Hook. \& Arn., J. Bot. (Hooker) 1: 293. 1834.

Metastelma diffusum (Gillies ex Hook. \& Arn.) Decne., DC. Prodr. 8: 516.1844.

Astephanus streptocarpus E. Fourn., Ann. Sci. Nat., Bot. 6 (14): 367 1882. Tipo: PARAGUAY. In planitie Doña Juana, 18-IX-1874, B. Balansa 1366 (Holotipo P!, isotipo G!). syn. nov.

El protólogo de Astephanus streptocarpus está reducido a 5 líneas, con sólo la descripción de hojas y fruto; con ello y sobre la base del examen del tipo y de nuevas colecciones la consideramos como un sinónimo de Orthosia virgata.

Esta especie vive en Argentina, Uruguay y Paraguay, y en Brasil está restringida a los estados de Rio Grande do Sul, Paraná y Santa Catarina. Habita bosques, selvas en galería, alto de montañas y cuestas rocosas.

Material estudiado: BRASIL. Paraná: Piraquara, s. data, Donbrowski \& Scherer Neto 7472 (R). Rio Grande do Sul: São Francisco de Paula, Flona, 15II-2008, Seger 871 (R). PARAGUAY. Amambay: In altaplanitie, Sierra de Amambay, VIII-1912/13, Hassler 11299 (G). Paraguarí: Bords de Mbay, entre Paraguari et Valenzuela, 23-VII-1883, Balansa 4642 (G). ibidem: Prope Sapucay, IX, s. col. (G).

Metastelma parviflorum (Sw.) Schult., Syst. Veg. 6: 120. 1820.

Cynanchum parviflorum Sw., Prodr. 53. 1788. Tipo: India occidentalis, Plum. ic. 215. f. 1. Sinonimia véase Silva \& Fontella-Pereira (1977) y Liede \& Meve (2004, sub Metastelma schlechtendalii Decne.).

Metastelma tubatum Griseb., Abh. Königl. Ges. Wiss. Göttingen 24: 227. 1879. Tipo: ARGENTINA. Salta, cerca del pasaje del Río Juramento, 16-21-III-1873, P. G. Lorentz \& G. H. E. W. Hieronymus 319 (Lectotipo GOET [GOET000729], aquí designado, paratipo GOET [GOET000725]). syn. nov.

En el protólogo de $M$. tubatum, el tipo está indicado de la siguiente manera: "S.: ad fl. Juramento". En el herbario GOET existen dos colectas hechas por P. G. Lorentz, \& G. H. E. W. Hieronymus de números 313 (GOET000725) y 319 (GOET000729), siendo éste último elegido como lectotipo. Escogemos este material teniendo en vista que Grisebach trabajó en Göttingen y que el mismo concuerda con la descripción del protólogo, aunque la colección 313 podría también haber sido escogida ya que hay pocas diferencias entre estos materiales. En este trabajo citamos a Metastelma parviflorum (Sw.) Schult. para Paraguay y Argentina (nuevos registros), que fue tratada por Meyer (1944) como Metastelma tubatum ilustrándola con una lámina bellísima.

Metastelma parviflorum tiene una distribución geográfica amplia, desde el sur de los Estados Unidos, México, América Central y norte de América del Sur, habitando también en Paraguay y 


\section{J. N. Fontella et al. - Notas taxonómicas en Asclepiadoideae}

Argentina. Crece generalmente en suelos lateríticos de coloración roja, habitando matorrales, bosques secos, bosque secundarios, en bordes de bosque, cuestas, escarpas y colinas.

Liede \& Meve (2004) en la revisión de Metastelma, redescribieron a Metastelma schlechtendalii, incluyendo nuevos sinónimos, nuevas variedades y además ampliaron su distribución geográfica. Sin embargo, Silva \& Fontella-Pereira (1977), sobre la base del examen de los "tipos" de $M$. parviflorum (Sw.) Schult. y $M$. schlechtendalii, además de numerosos ejemplares estudiados, ya habían considerado a $M$. schlechtendalii como sinónimo de M. parviflorum, aquí aceptado como el nombre más correcto.

Material estudiado: MEXICO. Vera Cruz: alt. 3000 pds, Galeoti 1541 ( G). BAHAMAS. Eleuthera Island: Governor's Harbor, 0,5 miles N of Spanish Main, 31-XII-1968, Lewis 7217 (NY). PUERTO RICO. Coamo: Bo., Pasto, Rt $155 \mathrm{Km}$ 6,8, at base of cliff along $0,5 \mathrm{~km}$ stretch of road, $18^{\circ} 06^{\prime} 84^{\prime \prime} \mathrm{N}, 66^{\circ}$ 22' 84" W, 30-XI-2002, Axelrod \& Axelrod 12363 (CTES). ISLAS VÍRGENES DE LOS ESTADOS UNIDOS. Saint Croix: Arm' as Hope, 2-X-1925, Thompson 932 (NY). GUATEMALA. St. Thomas, 1841, Friedrichsthal s.n. (NY). SAN BARTOLOMÉ. Gustavia: 27-XI-1934 (NY). ANTÍGUA Y B ARBUDA. Near St. Mary's, 10.XI.1913, Rose et al. 3396 (NY). GUADALUPE. In planitiebus insulae Guadeloupe, 1845, Funck \& Schlim 14 (LE). MARTINICA. Bord de la Mer prés Fort de France, 1867-1970, Hahn 739 ( NY). TRINIDAD Y TOBAGO. Monos: XI-1915, Bradway 7428 (NY). VENEZUELA. Camburi chiquito, 9-VI-1917, Curan \& Haman 838 ( NY). GUYANA. Road from Karanambo to Yupukari, 26IX-1988, Maas et al. 7675 (CTES). BRASIL. Amazonas: Margens do alto Rio Branco, IX-1913, Kublmann 605 (RB). PARAGUAY. Alto Paraguay: Picada al $\mathrm{N}$ de la línea 3 y de la pista de aviación en dirección a Cerro Cabrera, $19^{\circ} 40^{\prime} \mathrm{N}, 61^{\circ} 42^{\prime} \mathrm{W}$, 28-X-1992, Fortunato et al. 3664 (BAB, CTES, FCQ). Boquerón: Fortín Gabino Mendoza, a $12 \mathrm{~km}$ del desvío a Parque Cué sobre Ruta a Lagerenza, 2303'58'S, 61³7'15’W, 30-III-1995, Degen \& Mereles 3270 (FCQ). Nueva Asunción: Ruta-Trans-

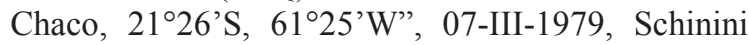
16390 (CTES). ARGENTINA. Salta: Rivadavia, entre Dragones y Pluma de Pato, alt. 240 m s.m., 12III-1992, Toledo \& Neumann 10780 (CTES).
Metalepis cubensis (A. Rich.) Griseb., Cat. Pl. Cub. 160. 1866.

Gonolobus cubensis A. Rich., Fl. Cub. Fanerog. 2: 98.1845. Tipo: CUBA, Crescit in Vuelta de abajo (J. Maria Valenzuela), R. de la Sagra s.n. (Holotipo P!). Roulinia ekmanii Malme, Ark. Bot. 16(15): 18. 1920. Tipo: ARGENTINA. Misiones, Posadas, Bonpland, 9-I-1908, E. L. Ekmann 406 (Holotipo S!). syn. nov. Telminostelma ekmanii (Malme) Fontella \& E. A. Schwarz, Bol. Mus. Bot. Munic. Curitiba 45: 5. 1981. syn. nov.

Cynanchum ekmanii (Malme) Sundell, Evol. Monogr. 5: 37. 1981. syn.nov.

Fontella-Pereira \& Goes (2009) adoptaron los conceptos genéricos de Grisebach (1866), Urban (1919) y Morillo (1997) para Metalepis, enumerando en esa ocasión varios sinónimos. Los mismos autores consideraran al género como distinto de Cynanchum, relacionándolo, por otro lado, con Matelea Aubl. y Gonolobus Michx., pues como estos últimos, Metalepis presenta lóculos dispuestos en el borde apical del ginostegio y caudículas dispuestas horizontalmente también en el borde del ginostegio.

Morillo (1997) reporta al género Metalepis para el sur de México, Cuba, Tobago, Colombia, Venezuela, Guyana Francesa, Ecuador y Sudeste de Brasil (Rio de Janeiro). Con la inclusión de Roulinia ekmannii en la sinonimia de Metalepis cubensis, la distribución del referido taxón se incrementa, extendiéndose desde América Central hasta el sur de Brasil (Estado do R io Grande do S ul), Paraguay y Argentina. La especie había sido descripta e ilustrada en Meyer, Lilloa 23: 57, fig. 4. 1950, como Roulinia ekmanii.

\section{AgRADECIMIENTOS}

Los autores agradecen a 1 os curadores de los herbarios B, BAB. BACP, CTES, FCQ, G, JE, K, LE, M, NY, P, RB, S, SI, W, WU, que gentilmente pusieron a disposición ejemplares de herbario e imágenes digitales que permitieron llevar a cabo este trabajo; al Consejo Nacional de Desenvolvimiento Científico e Tecnológico (CNPq) de Brasil (Proyecto N. 303929/2009-0) por la ayuda al primer autor. 


\section{Bibliografía}

AUBLET. 1775. Matelea. In: Hist. Pl. Guiane 1: 277-278, pl. 109, f. 1.

BORNMUELLER, J. 1934. Asclepiadaceae. In: Florula Riograndensis. Bearbeitung der von Alfred Bornmueller den Jahren 1903-1907 gesammelten Pflanzen. Revista Sudamer. Bot. 2: 3-4.

EZCURRA, C., J. FONTELLA-PEREIRA \& L. S. KINOSHITA. 2008. Apocynaceae. In: Zuloaga, F. O., O. Morrone \& M. J. Belgrano (eds.). Catálogo de las Plantas Vasculares del Cono Sur. Vol. 2. Monogr. Syst. Bot. Missouri Bot. Gard. 107: 1090-1143.

FONTELLA-PEREIRA, J. \& M. B. de GOES, 2009. Asclepiadoideae (Apocynaceae) do Sudeste do Brasil-II. Novos sinônimos e novas ocorrências para Metalepis cubensis no Brasil. Pesqui. Florest. Brasil. 58: 75-78.

FOURNIER, E. 1885. Asclepiadaceae. in: Martius, C. F. P. (ed.), Flora Bras. 6: 189-332.

GOYDER, D. J. 2006. Rojasia reinstated and new names and combinations in Matelea (Apocynaceae: Asclepiadoideae). Kew Bull. 61: 31-33.

GRISEBACH, A. 1866. Asclepiadaceae. in: Catalogus plantarum cubensium. Lipsiae p. 179-180.

MEYER, T. 1944. Asclepiadaceae. in: H. R. DESCOLE (ed.), Gen. Sp. Pl. Argent. 2: 1273.
LIEDE, S. \& U. MEVE. 2004. Revision of Metastelma (Apocynaceae-Asclepiadoideae) in southwestern North America and Central America. Ann. Missouri Bot. Gard. 91: 3186.

MEYER, T. 1970. Asclepiadaceae argentinenses novae aut criticae, V. Lilloa 33: 69-84.

MALME, G. O. A. 1932. Asclepiadaceae austroamericanae praecipue andinae. Ark. Bot. 25A: 1-26.

MORILLO, G. 1984. Nuevas especies, nuevos nombres y nuevas combinaciones en Matelea Aubl. Ernstia 24: 35-40.

MORILLO, G. 1997. Revisión preliminar de Metalepis Griseb. (Asclepiadaceae). Pittieria 26: 65-99.

RAPINI, R., R. MELLO-SILVA \& M. K. KAVASAKI. 2001. Asclepiadoideae (Apocynaceae) da Cadeia do Espinhaço de Minas Gerais, Brasil. Bol. Bot. Univ. São Paulo 19: 55-169.

SILVA, N. M. F. DA \& J. FONTELLA-PEREIRA. 1977. Estudos em Asclepiadaceae, IX. Sobre a identidade de Acrocoryne caribaea Turcz. Bol. Mus. Bot. Mun. 30: 1-11.

URBAN, A. I. 1919. Sertum antillanum. VIII. Feddes Repert. Spec. Nov. Regni Veg. 16: 3241.

Recibido el 26 de noviembre de 2013, aceptado el 1 de julio de 2014 . 